http://jmscr.igmpublication.org/home/ ISSN (e)-2347-176x ISSN (p) 2455-0450 crossref DOI: https://dx.doi.org/10.18535/jmscr/v8i9.27

\title{
Comparing long-term outcome of Traumatic Brain Injury by impact score in the ED with Glasgow outcome score
}

\author{
Authors \\ Dr Vernica Kala ${ }^{*}$, Dr Suraj Horakeri ${ }^{2}$, Dr Sagar Sinha ${ }^{3}$, Dr Dattatray Bhusare ${ }^{4}$ \\ ${ }^{1,2}$ Second- year MD Resident, Emergency Medicine \\ ${ }^{3}$ Assistant Professor, Emergency Medicine \\ ${ }^{4}$ Head of Department, Emergency Medicine \\ MGM Medical College and Hospital, Navi Mumbai, India \\ *Corresponding Author
}

Dr Vernica Kala

\section{Introduction}

- Traumatic brain injury has been associated with severe outcomes including vegetative states, significant disability, residual neurological deficits and assisted living. These have a major impact on quality of life of head trauma survivors. Unfavourable outcomes are routinely assessed by the Glasgow Outcome Scale (GOS), but it is not a prognostic score. Prognostic models are useful for several reasons. Clinically, they help doctors make better decisions about treatment. IMPACT SCORE (International Mission for Prognosis and Analysis of Clinical Trials in TBI) is a prognostic score to predict the long-term outcome in TBI. IMPACT score was developed by impact research group over the past several years using a large database that includes data from eight randomized controlled trials and three observational studies. This score will be calculated in an emergency room in Indian setting by using clinical parameters. This score will be correlated with short term morbidity outcomes and long-term outcome by the Glasgow outcome score. Short term morbidity will be assessed by;

- Need for ventilation in first five days

- Need for Operative treatment in the first five days

- Need for ICU stay for 5 or more days

- Presence of seizure in first 5 days

- Nausea vomiting in first 5 days

Long term outcome will be assessed by the Glasgow outcome score.

\section{Aim and Objective of the Study}

1. To calculate the impact score based on clinical parameters in Emergency room and to prove that it helps in estimating long term outcome after traumatic brain injury.

2. Comparing impact score results with morbidity markers and Glasgow Outcome Scale. 


\section{Methodology}

All patients who presented to the ED with TBI over a 1-month period were included in the study. These patients will be followed-up for total 3months and relevant data with a multivariate analysis will be determined to validate the impact score. This score includes predictive characteristics-age, motor score, pupillary reactivity, hypoxia, hypotension, positive CT classification finding, presence of $\mathrm{SAH}$, presence of EDH. Each patient got a score out of 22 (22 being the most severe) based on this system. Independent assessment of this score will be done for correlation with morbidity parameters. These patients will also be evaluated by the Glasgow Outcome Scale. Finally, the impact score will be compared with the Glasgow Outcome Scale to prove that clinical parameters in the emergency room can help in assessing long term outcomes.

Sample Size- 10

\section{Inclusion Criteria}

1) Age group (>18years).

2) All patients presenting to the ED with traumatic brain injury.

\section{Exclusion Criteria}

1) Children (<18years).

2) Not a patient of traumatic brain injury.

On Admission (In Emergency Room): proforma:

Table No 1: Impact score

\begin{tabular}{|c|c|c|l|l|l|}
\hline CHARECTERSTICS & VALUE & SCORE & TOTAL SUM & & \\
\hline 1) AGE & $<30$ & 0 & & & \\
\hline & $30-39$ & 1 & & & \\
\hline & $40-49$ & 2 & & & \\
\hline & $50-59$ & 3 & & & \\
\hline & $60-69$ & 4 & & & \\
\hline & $70+$ & 5 & & & \\
\hline 2)MOTOR SCORE & none & 6 & & & \\
\hline & abnormal flexion & 4 & & & \\
\hline & Normal flexion & 2 & & & \\
\hline & Localises/obeys & 0 & & & \\
\hline & Untestable/missing & 3 & & & \\
\hline 3)PUPILLARY REACTIVITY & Both pupil reactive & 0 & & & \\
\hline & One pupil reactive & 2 & & & \\
\hline & No pupil reactive & 4 & & & \\
\hline 4) HYPOXIA & yes & 1 & & & \\
\hline & no & 0 & & & \\
\hline 5)HYPOTENSION & yes & 2 & & & \\
\hline & no & 0 & & & \\
\hline 6) CT CLASSIFICATION & 1 & -2 & & & \\
\hline & 2 & 0 & & & \\
\hline & $3 / 4$ & 2 & & & \\
\hline & $5 / 6$ & 2 & & & \\
\hline 7) SAH & yes & 2 & & & \\
\hline & no & 0 & & & \\
\hline 8) EDH & yes & -2 & & & \\
\hline & no & 0 & & & \\
\hline
\end{tabular}

Table No 2: Morbidity Markers

\begin{tabular}{|l|c|c|}
\hline PARAMETERS & Yes/no & score \\
\hline Icu stay more than 5 days & & \\
\hline Need for ventilation & & \\
\hline Need for operative intervention & & \\
\hline Seizure & & \\
\hline Vomiting/Giddiness & \\
\hline \\
Total Score
\end{tabular}


Assessment after 3 Months

Glasgow Coma Outcome Score: -

$/ 5$

\begin{tabular}{|l|c|c|}
\hline SCORE & CATEGORY & INTERPRETATION \\
\hline 1 & DEAD & DEAD \\
\hline 2 & VEGETATIVE STATE & ABSENCE OF AWARENESS OF SELF AND ENVIRONMENT \\
\hline 3 & SEVERE DISABLITY & NEEDS FULL TIME ASSISTANCE \\
\hline 4 & MODERATE DISABILTY & INDEPENDENT, BUT CANNOT RESUME OR PARTLY RESUME \\
& & WORK/SOCIAL ACTIVITIES \\
\hline 5 & GOOD RECOVERY & $\begin{array}{c}\text { FULL RECOVERY /MINOR PHYSICAL OR MENTAL DEFICIT THAT } \\
\text { DOES NOT AFFECT DAILY LIFE }\end{array}$ \\
\hline
\end{tabular}

\section{Observation}

Table No 3

\begin{tabular}{|c|c|c|c|c|c|c|}
\hline $\begin{array}{l}\text { TOTAL } \\
\text { SCORE } \\
\text { (out of 22) } \\
\end{array}$ & $\begin{array}{l}\text { ICU STAY } \\
>5 \text { DAYS }\end{array}$ & $\begin{array}{l}\text { NEED } \\
\text { FOR } \\
\text { VENTI }\end{array}$ & $\begin{array}{l}\text { NEED } \\
\text { FOR OT }\end{array}$ & SEIZURE & $\begin{array}{c}\text { VOMITING/GID } \\
\text { DINESS }\end{array}$ & $\begin{array}{c}\text { GLASGOW } \\
\text { OUTCOME SCALE } \\
\text { SCORE }\end{array}$ \\
\hline 3 & $\mathrm{NO}, 0 / 1$ & $\mathrm{NO}, 0 / 1$ & $\mathrm{NO}, 0 / 1$ & $\mathrm{NO}, 0 / 1$ & YES, $1 / 1$ & SCORE 5 \\
\hline 2 & $\mathrm{NO}, 0 / 1$ & $\mathrm{NO}, 0 / 1$ & $\mathrm{NO}, 0 / 1$ & $\mathrm{NO}, 0 / 1$ & YES, 1`/1 & SCORE 4 \\
\hline 12 & YES, 1/1 & YES, 1/1 & YES, $1 / 1$ & $\mathrm{NO}, 0 / 1$ & YES, 1/1 & SCORE 2 \\
\hline 3 & $\mathrm{NO}, 0 / 1$ & $\mathrm{NO}, 0 / 1$ & $\mathrm{NO}, 0 / 1$ & $\mathrm{NO}, 0 / 1$ & YES, $1 / 1$ & SCORE 5 \\
\hline 2 & $\mathrm{NO}, 0 / 1$ & $\mathrm{NO}, 0 / 1$ & $\mathrm{NO}, 0 / 1$ & $\mathrm{NO}, 0 / 1$ & $\mathrm{NO}, 0 / 1$ & SCORE 5 \\
\hline 2 & $\mathrm{NO}, 0 / 1$ & $\mathrm{NO}, 0 / 1$ & $\mathrm{NO}, 0 / 1$ & NO, $0 / 1$ & YES, 1/1 & SCORE 5 \\
\hline 9 & YES, $1 / 1$ & $\mathrm{NO}, 0 / 1$ & $\mathrm{NO}, 0 / 1$ & YES, 1/1 & YES, $1 / 1$ & SCORE 3 \\
\hline 15 & YES, 1/1 & YES, 1/1 & $\mathrm{NO}, 0 / 1$ & $\mathrm{NO}, 0 / 1$ & YES,1/1 & SCORE 2 \\
\hline 15 & YES, $1 / 1$ & YES, 1/1 & YES, $1 / 1$ & $\mathrm{NO}, 0 / 1$ & YES, $1 / 1$ & SCORE 2 \\
\hline 3 & $\mathrm{NO}, 0 / 1$ & $\mathrm{NO}, 0 / 1$ & $\mathrm{NO}, 0 / 1$ & $\mathrm{NO}, 0 / 1$ & YES, 1/1 & SCORE 5 \\
\hline
\end{tabular}

Statistical Analysis

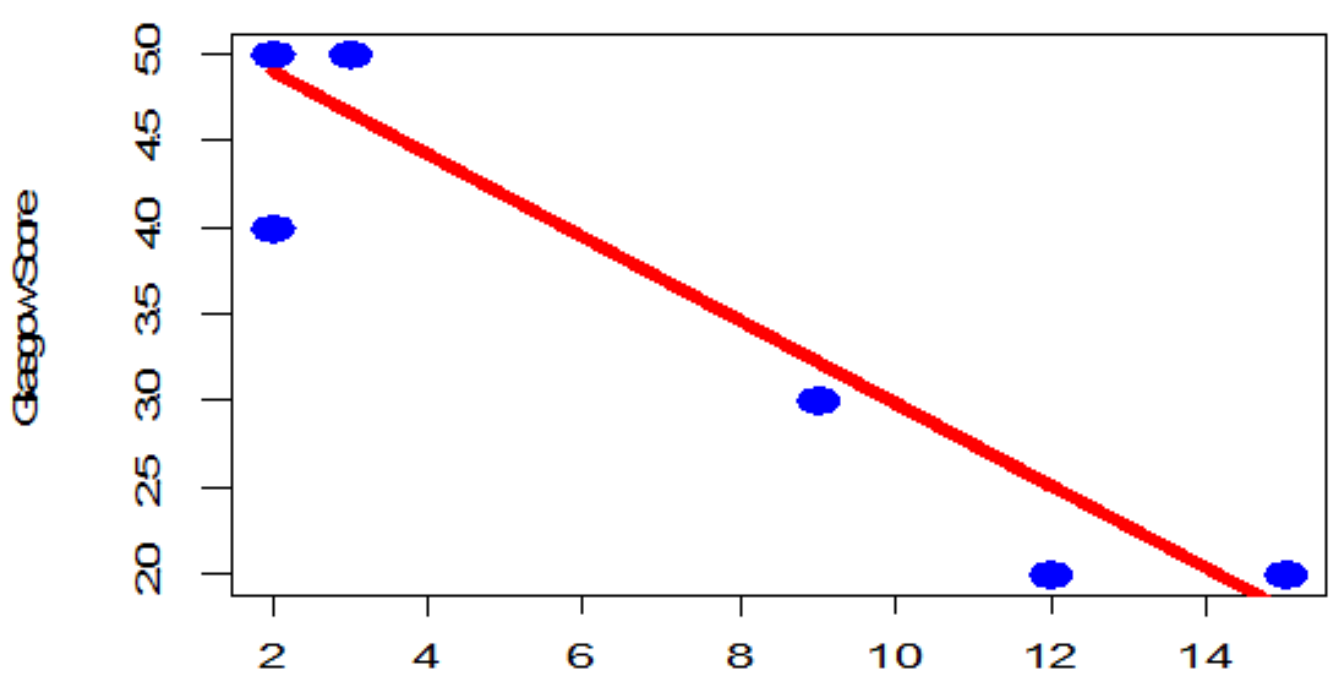

Total Score

Figure 1: Interpretation: The correlation value of -0.954 with p-value, 0.05 indicates significant negative correlation between Total IMPACT Score and Glasgow score. Higher the total impact score lower is the Glasgow score. Regression analysis is used to develop the predictive model. 


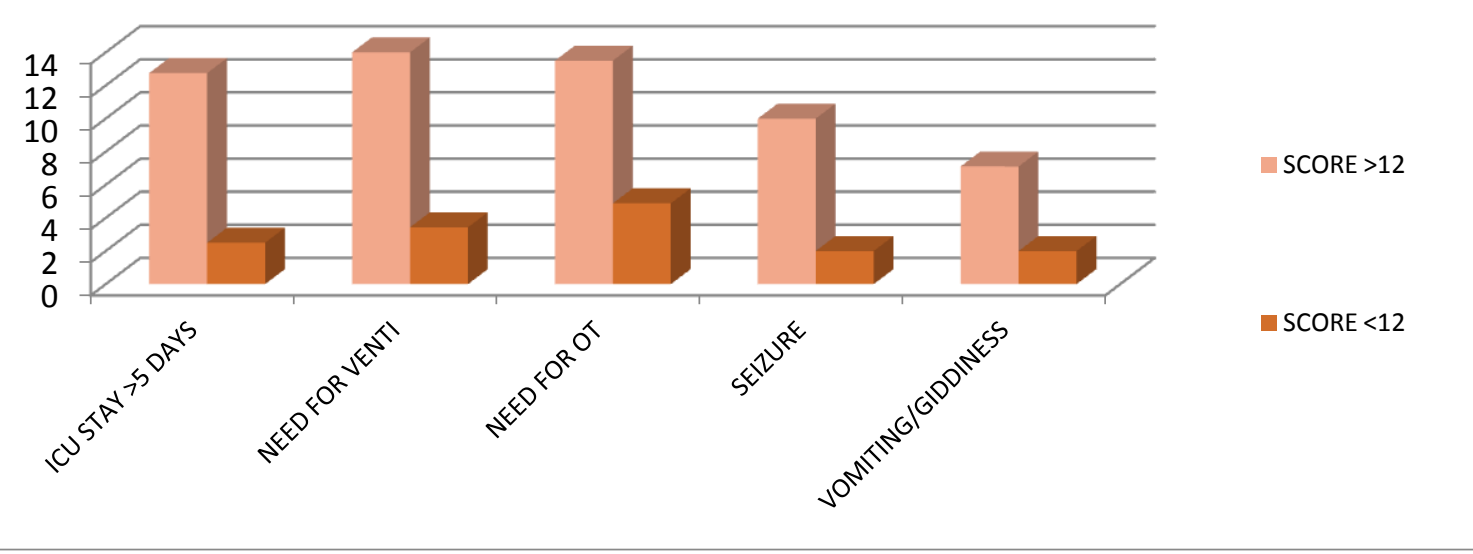

Figure 2: Correlation between Prognostic Impact Score and Mortality Markers

\section{Result}

- All predictors used in impact score had statistically significant associations with 3 -month Glasgow coma scale.

- Since p-value for impact score is significant, it indicates that impact score can be used to predict Glasgow outcome score significantly. adjusted $\mathrm{r}$ square of 0.8995 indicates that impact score alone can explain variation in Glasgow outcome scale almost up to $89.95 \%$. the model can be written as below:

Glasgow outcome score $=5.38362-$ 0.23994 (impact score)

- IMPACT score made using ED parameters correlates with the mortality markers.

\section{Conclusion and Discussion}

- Early clinical parameters in the emergency room can be used to predict long-term outcome.

- The impact score discriminated adequately between patients with poor and good outcomes.

- prognostic scoring tools can be used by emergency physicians to take better treatment/clinical decisions, so early assessment in the emergency room is very important in a case of traumatic brain injury.

\section{References}

1. Teasdale G, Jennett B (1974) Assessment of coma and impaired consciousness. A practical scale. Lancet 2: 81-84.

2. Jennett B, Bond M (1975) Assessment of outcome after severe brain damage. Lancet 1: 480-484.

3. Jennett B, Teasdale G, Braakman R, Minderhoud J, Knill-Jones R (1976) Predicting outcome in individual patients after severe head injury.

4. Hernandez AV, Steyerberg EW, Taylor GS, Marmarou A, Habbema JD, et al. (2005) Subgroup analysis and covariate adjustment in randomized clinical trials of traumatic brain injury: a systematic review. Neurosurgery 57: 1244-1253.

5. Maas AI, Marmarou A, Murray GD, Teasdale SG, Steyerberg EW (2007) Prognosis and clinical trial design in traumatic brain injury: the IMPACT study. J Neurotrauma 24: 232-238.

6. Murray GD, Butcher I, McHugh GS, Lu J, Mushkudiani NA, et al. (2007) Multivariable prognostic analysis in traumatic brain injury: results from the IMPACT study. J Neurotrauma 24: 329337. 